\title{
ON THE SOLID HULLS OF THE NEVANLINNA AND SMIRNOV CLASSES
}

\author{
Marek Nawrocki \\ A. Mickiewicz University, Faculty of Mathematics and Computer Science \\ ul. Umultowska 87, 61-614 Poznań, Poland; nawrocki@amu.edu.pl
}

\begin{abstract}
In the paper, the solid and positive solid hulls of the Nevanlinna class and the Smirnov class of the unit disc are described. The result is applied to find the best possible estimations of the Taylor coefficients and the multipliers from the Smirnov class into some large spaces of holomorphic functions. These results provide a much "softer" and easier way to obtain even stronger results on the multipiers and the mean growth of the Taylor coefficients proved by Yanagihara.
\end{abstract}

\section{Introduction}

In this paper we denote by $\omega$ the topological vector space consisting of all complex sequences $x=(x(n)), n \in \mathbf{Z}_{+}=\{0,1,2, \ldots\}$, and equipped with the topology of coordinate-wise convergence. A subset $X$ of $\omega$ is said to be solid if $y \in X$ whenever $y \in \omega$ and $|y| \leq|x|$ for some $x \in X$. For each subset $X$ of $\omega$, the solid hull of $X$ defined as

$$
S(X)=\{y \in \omega:|y| \leq|x| \text { for some } x \in X\}
$$

is clearly the smallest solid subset $S(X)$ containing $X$.

To find the solid hull of a set $X \subset \omega$ we usually have to describe the strongest growth condition of absolute values of elements in $X$. This is often not an easy problem, especially when $X$ is a space of holomorphic functions on the unit disc D, each function $f \in X$ being identified with the sequence $(\hat{f}(n))$ of its Taylor coefficients. (Thus we are actually dealing with the subspace $\widehat{X}=\{\hat{f}: f \in X\}$ of $\omega$.) To illustrate this point, let us recall what is known in the case of the classical Hardy spaces $H^{p}=H^{p}(\mathbf{D})(0<p \leq \infty)$. Evidently, $S\left(H^{2}\right)=l^{2}$, but the other cases are not that simple. Thus also $S\left(H^{p}\right)=l^{2}$ for $2<p<\infty$ (by Littlewood's theorem on random power series [D, Thm. A.5]) as well as $S\left(H^{\infty}\right)=l^{2}$ (by a result of Kisliakov $[\mathrm{K}])$. Finally, for $0<p<1$, Jevtić and Pavlović [JP1] have recently proved that $S\left(H^{p}\right)$ is the space of all complex sequences $(x(n))$ such that

$$
\sum_{n=0}^{\infty}(n+1)^{p-2}\left(\sup _{0 \leq k \leq n}|x(n)|^{p}\right)<\infty .
$$

The case $1 \leq p<2$ is still open. The same authors have recently described the solid hulls of the Hardy-Lorenz spaces [JP2]. Moreover, Mastyło and Mleczko [MM]

doi:10.5186/aasfm.2014.3953

2010 Mathematics Subject Classification: Primary 30H15, 30H50, 46A16; Secondary 46E05.

Key words: Nevanlinna class, Smirnov class, Hardy algebra, Bergman algebra, Fréchet envelope, nuclear power series spaces.

The author is very grateful Lech Drewnowski for his remarks and comments on this material. 
constructed solid hulls for some quasi-Banach spaces of analytic functions which can by obtained by interpolation.

In this paper we describe the solid halls of the largest spaces in the scale of Hardy spaces, that is, the Nevanlinna class $N$ and the Smirnov class $N_{*}$ of the unit disc.

Recall that the Nevanlinna class $N=N(\mathbf{D})$ on the unit disc is the space of all holomorphic functions $f$ on $\mathbf{D}$ such that

$$
\|f\|=\sup _{r \in(0,1)} \int_{0}^{2 \pi} \log \left(1+\left|f\left(r e^{i t}\right)\right|\right) d t<\infty,
$$

and the Smirnov class $N_{*}=N_{*}(D)$ is the subspace of $N$ consisting of all functions $f$ such that the family $\left\{\log \left(1+\left|f\left(r e^{i t}\right)\right|\right): 0<r<1\right\}$ is uniformly integrable on $[0,2 \pi]$.

More generally, if $p>1 L H^{p}$ is the Hardy-Orlicz space consisting of all holomorphic functions $f$ on the unit disc such that

$$
\|f\|_{p}^{p}=\sup _{r \in(0,1)} \int_{0}^{2 \pi} \log ^{p}\left(1+\left|f\left(r e^{i t}\right)\right|\right) d t<\infty .
$$

Moreover, let $F_{*}^{p}$ denote the space of all complex sequences $x=(x(n))$ such that

$$
\|x\|_{p, c}=\sup _{n \in \mathbf{Z}_{+}}|x(n)| \exp \left(-c n^{\frac{1}{p+1}}\right)<\infty \quad \text { for each } c>0
$$

with the topology defined by the sequence of norms $\|\cdot\|_{p, 1 / k}, k=1,2, \ldots$

It is well known that each function in $N$ is a quotient of two functions in $H^{\infty}$ (see [D] Theorem 2.1), and that

$$
H^{\infty} \subset H^{p} \subset H^{q} \subset N_{*} \subset N \text { for } 0<q<p<\infty .
$$

In this paper we show that the solid hull of the Smirnov class can be identified with the space $F_{*}$ of all complex sequences $((x(n))$ such that $|x(n)|=O(\exp (c \sqrt{n})$ for each $c>0$ (Theorem 4.1). The space $F_{*}$ was invented by Yanagihara [Y1] while studying Fréchet envelope of $N_{*}$ (cf. [DRS]). The crucial points in Yanagihara's result where descriptions of the best possible mean growth of the Taylor coefficients of functions in $N_{*}$ and the multipliers from $N_{*}$ into $H^{\infty}$ (cf. [Y2]). Theorem 4.1 and Corollary 4.2 provide a much "softer" and easier way to obtain even stronger result on the mean growth of the Taylor coefficients. Theorem 4.1 is applied in Theorem 5.1 to describe multipliers from $N_{*}$ both into $H^{\infty}$ and some "large" algebras $L H^{p}, p \geq 1$, of holomorphic functions still in a soft and easy way.

\section{Positive solid hulls}

If $X$ is a vector subspace of $\omega$ then, in general, its solid hull $S(X)$ is not a linear set (it need not be closed under addition). Fortunately, for some applications it suffices to study solid hulls of the cone of nonnegative elements in the space.

For any subset $X$ of $\omega$ we denote

$$
X_{+}=\{x \in X: x \geq 0\} .
$$

Note that if $X$ is a linear subspace, then $X_{+}$is a convex cone in $X$.

Fact 2.1. If $X$ is a linear subspace of $\omega$, then $S\left(X_{+}\right)$is a solid linear subspace of $\omega$ containing $X_{+}$. 
Proof. Let $y_{j} \in S\left(X_{+}\right), \lambda_{j} \in \mathbf{C}, j=1,2$. Then $\left|y_{j}\right| \leq x_{j}$ for some $x_{j} \in X_{+}$, $j=1,2$. Since

$$
\left|\lambda_{1} y_{1}+\lambda_{2} y_{2}\right| \leq\left|\lambda_{1}\right|\left|y_{1}\right|+\left|\lambda_{2}\right|\left|y_{2}\right| \leq\left|\lambda_{1}\right| x_{1}+\left|\lambda_{2}\right| x_{2} \in X_{+},
$$

it follows that $\lambda_{1} y_{1}+\lambda_{2} y_{2} \in S\left(X_{+}\right)$.

In this paper, all vector spaces under consideration have natural "sequence space" group or vector topologies. Assume that $X$ is a vector subspace of $\omega$ equipped with a locally balanced group (vector) topology $\tau$. We say that $X=(X, \tau)$ is a sequence vector group (space) if

(S) $\tau$ is stronger than the topology of coordinate-wise convergence, i.e., the coefficient functionals $x \mapsto x(n)$ are continuous on $X$ for all $n \in \mathbf{Z}_{+}$,

and that it is a solid sequence group (space) if $X$ is solid and $\tau$ has a base of neighborhoods of zero consisting of solid sets.

Fact 2.2. Let $X=(X, \tau)$ be a sequence space with a group [vector] topology $\tau$, and let $\mathcal{U}$ be a base of $\tau$-neighborhoods of zero. Then the family

$$
\mathcal{U}_{+}(0)=\left\{S\left(U_{+}\right): U \in \mathcal{U}\right\}
$$

is a base of solid neighborhoods of zero for a solid group [vector] topology $\tau_{+}$on $S\left(X_{+}\right)$. Moreover, if $\tau$ is Hausdorff or metrizable, so is $\tau_{+}$, respectively.

Proof. To see the first part it is enough to observe that if $U, V \in \mathcal{U}$ and $V+V \subset U$, then $S\left(V_{+}\right)+S\left(V_{+}\right) \subset S\left(V_{+}+V_{+}\right) \subset S\left(U_{+}\right)$, and that $S\left(U_{+}\right)$is absorbing in $S\left(X_{+}\right)$ whenever $U$ is absorbing in $X$.

Assume $\tau$ is Hausdorff and suppose an element $y \in S\left(X_{+}\right)$is such that $x \in S\left(U_{+}\right)$ for all $U \in \mathcal{U}$. Thus for each $U \in \mathcal{U}$ one has $|y| \leq x_{U}$ for some $x_{U} \in U_{+}$. Then the net $\left(x_{U}\right)$ is $\tau$-convergent, hence also coordinate-wise convergent, to zero. It follows that $|y|=0$, and $\tau_{+}$is Hausdorff.

Finally, if $\tau$ is metrizable, then $\tau_{+}$is Hausdorff and has a countable base at zero, hence it is metrizable, too.

If a space $X=(X, \tau)$ as above is metrizable, then its topology is induced via the metric $d(x, y)=\|x-y\|$ by an $F G$-norm $\|\cdot\|$, i.e., a function $\|\cdot\|: X \rightarrow[0, \infty]$ which is subadditive, vanishes only at zero, and satisfies $\|\lambda x\| \leq\|x\|$ for all $x \in X$ and $|\lambda| \leq 1$. If, in addition, $\tau$ is a vector topology, then $\|\cdot\|$ can be chosen so that $\|\lambda x\| \rightarrow 0$ whenever $\lambda \rightarrow 0$ (i.e., $\|\cdot\|$ is an $F$-norm). Conversely, for every $F G$-norm $[F$-norm] $\|\cdot\|$ on $X$ the associated metric induces a locally balanced group [vector] topology on $X$. If the resulting metric space is complete then we call $X$ an $F G$-space [F-space].

We say that $\|\cdot\|$ is solid (monotone) if $|x| \leq|y|$ implies $\|x\| \leq\|y\|$.

Fact 2.3. Let $X=(X, \tau)$ be a sequence space and $\|\cdot\|$ an FG-norm inducing the topology $\tau$. Then the formula

$$
\|y\|=\inf \left\{\|x\|:|y| \leq x \in X_{+}\right\}
$$

defines a solid FG-norm inducing the topology $\tau_{+}$on $S\left(X_{+}\right)$. Moreover, if the space $X=(X,\|\cdot\|)$ is complete, so is the space $\left(S\left(X_{+}\right),\|\cdot\|\right)$.

Proof. It is easily seen that $\|\cdot\|$ is an $F G$-norm inducing $\tau_{+}$. 
In order to prove that $S\left(X_{+}\right)$is complete it suffices to show that if a sequence $\left(y_{j}\right)$ in $S\left(X_{+}\right)$is such that $\sum_{j=1}^{\infty}\left\|y_{j}\right\|<\infty$, then the series $\sum_{j=1}^{\infty} y_{j}$ is convergent in $S\left(X_{+}\right)$.

By the definition of $\|\cdot\| \cdot \|$, there is a sequence $\left(x_{j}\right) \subset X_{+}$such that $\left|y_{j}\right| \leq x_{j}$ and $\left\|x_{j}\right\| \leq 2\left\|y_{j}\right\|$ for each $j$. Then $\sum_{j=1}^{\infty}\left\|x_{j}\right\|<\infty$, hence by the completeness of $\|\cdot\|$ the series $\sum_{j=1}^{\infty} x_{j}$ converges unconditionally in $X$. Since $X$ is a sequence space, this series is unconditionally (hence absolutely) coordinate-wise convergent to some $y \in \omega$. Let $s_{J}=\sum_{j=J}^{\infty} y_{j}, J=1,2, \ldots$ Then $\left|s_{J}\right| \leq \sum_{j=J}^{\infty} x_{j}=: r_{J} \in X_{+}$, so $s_{J} \in S\left(X_{+}\right)$and $\left\|s_{J}\right\| \leq\left\|r_{J}\right\| \leq \sum_{j=J}^{\infty}\left\|x_{j}\right\| \rightarrow 0$ as $J \rightarrow \infty$. Finally, the series $\sum_{j=1}^{\infty} y_{j}$ is convergent in $S\left(X_{+}\right)$to $y=s_{1}$.

Fact 2.4. Let $X=(X, \tau)$ be a sequence vector group, $(Z, \zeta)$ a solid sequence group, and $T: \omega \rightarrow \omega$ a positive linear operator, i.e., $T x \geq 0$ whenever $x \geq 0$. If $T\left(X_{+}\right) \subset Z$, then also $T\left(S\left(X_{+}\right)\right) \subset Z$.

Proof. Take any $y \in S\left(X_{+}\right)$, so that $|y| \leq x$ for some $x \in X_{+}$. If $y$ is a real sequence, then $|T y|=\left|T y^{+}-T y^{-}\right| \leq T y^{+}+T y^{-}=T|y| \leq T x$. If $y$ is a complex sequence, then $y=u+i v$, where $u, v$ are real sequences, and

$$
|T y|=|T u+i T v| \leq|T u|+|T v| \leq T|u|+T|v| \leq T(2 x) .
$$

Since the space $Z$ is solid, we conclude that $T y \in Z$.

Remark 2.5. It is true, though not quite obvious, that $|T y| \leq T|y|$ for all $y=(y(n)) \in \omega$. To see this, consider first a positive linear functional $t$ on $\omega$. Then it is also continuous, hence of the form $t(y)=\sum_{j=0}^{k} a_{j} y(j)$, where $a_{1}, \ldots, a_{k} \in \mathbf{R}_{+}$. In consequence, $|t(y)| \leqslant \sum_{j=0}^{k} a_{j}|y(j)|=t(|y|)$. Now, if $T: \omega \rightarrow \omega$ is a positive linear operator, then its components $t_{n}\left(n \in \mathbf{Z}_{+}\right)$are positive linear functionals on $\omega$ and, by the preceding part, $|T y|=\left(\left|t_{n}(y)\right|\right) \leq\left(t_{n}(|y|)\right)=T|y|$.

\section{The solid hull of the Nevanlinna class}

Recall that

$$
\|f\|=\sup _{r \in(0,1)} \int_{0}^{2 \pi} \log \left(1+\left|f\left(r e^{i t}\right)\right|\right) d t
$$

is an $F G$-norm on the Nevanlinna class $N=N(\mathbf{D})$ and $(N,\|\cdot\|)$ is an $F G$-space. It is well known $[\mathrm{P}]$ that for each function $f \in N$,

$$
|\widehat{f}(n)|=O(\exp (O(\sqrt{n})) .
$$

In view of the lemma below, this estimate is best possible, and this fact will quickly lead us to the [positive] solid hull of $N$.

Lemma 3.1. (see $[\mathrm{P}]$, pp. 151-153) For each $c>0$ define

$$
f_{c}(z)=\exp \left(\frac{c}{2} \frac{1+z}{1-z}\right), \quad z \in \mathbf{D}
$$

Then

$$
f_{c} \in N, \quad\left\|f_{c}\right\|=\frac{c}{2} \quad \text { and } \quad \widehat{f}_{c}(n)=\exp (2 \sqrt{c n}[1+o(1)])
$$


Proposition 3.2. The solid hull $S(N)$ and the positive solid hull $S\left(N_{+}\right)$of the Nevanlinna class $N=N(\mathbf{D})$ coincide, and they are equal to the space $F$ consisting of all sequences $(x(n)) \in \omega$ such that

$$
\sup _{n}|x(n)| \exp (-c \sqrt{n})<\infty \quad \text { for some } c>0 \text {. }
$$

Proof. As it was mentioned above, $|\widehat{f}(n)|=O(\exp (O(\sqrt{n}))$ for each $f \in N$. Thus $N \subset F$ and so $S\left(N_{+}\right) \subset S(N) \subset F$ because $F$ is solid. By Lemma 3.1, for each sequence $x=(x(n)) \in F$ there are $C, c>0$ such that $|x(n)| \leq C \widehat{f}_{c}(n)$ for each $n \in \mathbf{Z}_{+}$. Consequently, $x \in S\left(N_{+}\right)$and finally $S\left(N_{+}\right)=S(N)=F$.

\section{The solid hull of the Smirnov class}

Recall that the Smirnov class $N_{*}=N_{*}(\mathbf{D})$ is the largest linear subspace of the Nevanlinna class which is a topological vector space in the relative topology (cf. [SS]). Moreover, $\|f-f(r \cdot)\| \rightarrow 0$ for each $f \in N_{*}$, where $f(r \cdot)(z)=f(r z)$ for $|z|<1 / r$.

Theorem 4.1. The solid hull $S\left(N_{*}\right)$ and the positive solid hull $S\left(\left(N_{*}\right)_{+}\right)$of the Smirnov class $N_{*}=N_{*}(\mathbf{D})$ coincide, and they are equal to the space $F_{*}$ consisting of all sequences $(x(n))$ such that

$$
\sup _{n}|x(n)| \exp (-c \sqrt{n})<\infty \quad \text { for each } c>0 .
$$

Proof. It is well known that $N_{*} \subset F_{*}$ (cf. [Y1, Theorem 1]). Consequently, $S\left(\left(N_{*}\right)_{+}\right) \subset S\left(N_{*}\right) \subset F_{*}$ since $F_{*}$ is solid. In order to prove that $F_{*} \subset S\left(\left(N_{*}\right)_{+}\right)$, fix $x=(x(n)) \in F_{*}$. It suffices to show that the series $\sum_{n=0}^{\infty} x(n) e_{n}$ (which converges to $x$ coordinate-wise) satisfies the Cauchy condition with respect to the $F$-norm $\|\cdot\|$ of the F-space $S\left(\left(N_{*}\right)_{+}\right)$(cf. Fact 2.3, (ASF)).

Take any $c>0$ and consider the function $f_{c}$ as in lemma 3.1. Then $\widehat{f}_{c}(n) \geq$ $d \exp (d \sqrt{n})$ for some $d>0$ and each $n \in \mathbf{Z}_{+}$. Since $x \in F_{*}$, there is $M \in \mathbf{Z}_{+}$such that $|x(n)| \leq d \exp (d \sqrt{n})$ for each $n \geq M$.

Now, fix arbitrary $k \geq j \geq M$, and set $P_{j, k}(z)=\sum_{n=j}^{k} z^{n}$. Since $\left\|f_{c}\right\|=\frac{1}{2} c$, there is $\delta>0$ so small that $\left\|f_{c}\right\|+\left\|\delta P_{j, k}\right\|<c$. Next, since $f_{c}(r \cdot) \rightarrow f_{c}($ as $r \rightarrow 1)$ uniformly on compact subsets of $\mathbf{D}$, we have $\sup _{r \in(0,1)}\left\|f_{c}(r \cdot)\right\|=\left\|f_{c}\right\|$. Moreover, $\widehat{f_{c}(r \cdot)}(n)=\widehat{f}_{c}(n) r^{n}>0$, hence we can find $r \in(0,1)$ such that $\widehat{f}_{c}(n) \leq \widehat{f_{c}(r \cdot)}(n)+\delta$ for $j \leq n \leq k$. Then the function $g=f_{c}(r \cdot)+\delta P_{j, k}$ belongs to $\left(N_{*}\right)_{+}$,

$$
\|g\| \leq\left\|f_{c}(r \cdot)\right\|+\left\|\delta P_{j, k}\right\| \leq\left\|f_{c}\right\|+\left\|\delta P_{j, k}\right\|<c,
$$

and

$$
|x(n)| \leq d \exp (d \sqrt{n}) \leq \widehat{f}_{c}(n) \leq \widehat{f_{c}(r \cdot)}(n)+\delta=\widehat{g}(n) \quad \text { for } j \leq n \leq k .
$$

It follows that $\left\|\sum_{n=j}^{k} x(n) e_{n}\right\|<c$. Consequently, the series $\sum_{n=1}^{\infty} x(n) e_{n}$ is Cauchy in $S\left(\left(N_{*}\right)_{+}\right)$.

It is easily seen that $F_{*}$ coincides with the space of all complex sequences $((x(n))$ such that $|x(n)|=O\left(\exp \left(c_{n} \sqrt{n}\right)\right.$ for some sequence of positive numbers $\left(c_{n}\right)$ tending to zero.

As an immediate consequence of this observation and Theorem 4.1 we now may state the following. 
Corollary 4.2. For any sequence $\left(c_{n}\right)$ of positive numbers tending to zero, there is a function $f \in\left(N_{*}\right)_{+}$such that

$$
\widehat{f}(n) \geq \exp \left(c_{n} \sqrt{n}\right) \quad \text { for each } n \in \mathbf{Z}_{+} .
$$

Remark 4.3. It follows that the estimate $|\widehat{f}(n)|=O(\exp (o(\sqrt{n}))$ for functions $f \in N_{*}$ is best possible in a very strong way. So far it was only known that for each positive sequence $\left(c_{n}\right)$ tending to zero there is a function $f \in N_{*}$ such that $|\widehat{f}(n)| \neq O\left(\exp \left(c_{n} \sqrt{n}\right)\right)[\mathrm{Y} 3, \mathrm{ST}]$.

\section{Multipliers}

Suppose that $X$ and $Y$ are sequence spaces, and let $\llbracket X, Y \rrbracket$ denote the collection of all sequences $\lambda=(\lambda(n))$ in $\mathbf{C}$ such that $\lambda x=(\lambda(n) x(n))$ belongs to $Y$ whenever $(x(n))$ is a member of $X$. This is a linear space whose elements are called the (coefficient) multipliers from $X$ into $Y$. By the Closed Graph Theorem, each $\lambda \in$ $\llbracket X, Y \rrbracket$ gives rise to a continuous linear operator

$$
\Lambda: X \rightarrow Y: x \mapsto \lambda x .
$$

Yanagihara [Y1] described the space of multipliers from the Smirnov class $N_{*}$ into the disc algebra $A=A(\mathbf{D})$ and the Hardy classes $H^{p}$ for $0<p \leq \infty$. Later, Jarchow, Montesinos, Wirths, and Xiao [JMWX] extended this result to the case of multipliers from the Nevanlinna algebras $L H^{p}$ into "small" spaces like $H^{p}$ above. The crucial point in the proofs of these results was that the topology of the space $Y$ in question is defined by a norm or a $q$-norm for some $0<q \leq 1$. The solid hull described in Theorem 4.1 allows us to find multipliers into "large spaces" $F_{*}^{p}$ which natural topology is not locally bounded. It also seems to provide a much "softer" and easier way to obtain the result than the earlier method used by Yanagihara.

Recall that $L H^{p}$ is the Hardy-Orlicz space consisting of all holomorphic functions $f$ on the unit disc such that

$$
\|f\|=\sup _{r \in(0,1)} \int_{0}^{2 \pi} \log ^{p}\left(1+\left|f\left(r e^{i t}\right)\right|\right) d t<\infty .
$$

Moreover, let $F_{*}^{p}$ denote the space of all complex sequences $x=(x(n))$ such that

$$
\|x\|_{p, c}=\sup _{n \in \mathbf{Z}_{+}}|x(n)| \exp \left(-c n^{\frac{1}{p+1}}\right)<\infty \quad \text { for each } c>0
$$

with the topology defined by the sequence of norms $\|\cdot\|_{p, 1 / k}, k=1,2, \ldots$

Theorem 5.1. For every sequence $\lambda=(\lambda(n))$ in $\mathbf{C}$, the following are equivalent:

(i) $\lambda(n)=O(\exp (-c \sqrt{n}))$ for some $c>0$.

(ii) $\lambda \in \llbracket N_{*}, A \rrbracket$.

(iii) $\lambda \in \llbracket N_{*}, H^{q} \rrbracket$ for some [all $0<q \leq \infty$.

(iv) $\lambda \in \llbracket N_{*}, L H^{p} \rrbracket$ for some [all $1<p<\infty$.

(v) $\lambda \in \llbracket N_{*}, F_{*}^{p} \rrbracket$ for some [all $1<p<\infty$.

(vi) $\lambda \in \llbracket F_{*}, F_{*}^{p} \rrbracket$ for some [all $] 1<p<\infty$.

Proof. The equivalences (i) $\Longleftrightarrow$ (ii) $\Longleftrightarrow$ (iii) were proved by Yanagihara [Y2], the implication (i) $\Longrightarrow$ (ii) being an easy part of the proof. Since $A \subset H^{q} \subset L H^{p} \subset$ $F_{*}^{p}$ for $0<q \leq \infty$ and $p>1$, so (ii) $\Longrightarrow$ (iii) $\Longrightarrow$ (iv) $\Longrightarrow$ (v) (cf. [ST] for the inclusion $\left.L H^{p} \subset F_{*}^{p}\right)$. 
Suppose now that $\lambda \in \llbracket N_{*}, F_{*}^{p} \rrbracket$ for some $1<p<\infty$. Since $F_{*}^{p}$ is solid, also $|\lambda|=(|\lambda(n)|) \in \llbracket N_{*}, F_{*}^{p} \rrbracket$. Now, the positive linear operator $\Lambda: x \mapsto|\lambda| x$ in $\omega$ maps $N_{*}$ into $F_{*}^{p}$, hence it also maps $S\left(\left(N_{*}\right)_{+}\right)=S\left(N_{*}\right)=F_{*}$ into $F_{*}^{p}$ (cf. Fact 2.4 and Theorem 4.1).

Finally assume that $\lambda \in \llbracket F_{*}, F_{*}^{p} \rrbracket$ for some $1<p<\infty$. Then the operator $\Lambda: F_{*} \rightarrow F_{*}^{p}: x \mapsto \lambda x$ is continuous, so there are $c>0$ and $\varepsilon>0$ such that $|\lambda(n) x(n)| \leq \exp \left(n^{\frac{1}{p+1}}\right)$ for each $x=(x(n)) \in F_{*}=F_{*}^{1}$ with $\|x\|_{1, c} \leq \varepsilon$. In particular, if $x_{n}=\varepsilon \exp (c \sqrt{n}) e_{n}$, then $x_{n} \in F_{*}$ and $\left\|x_{n}\right\|_{1, c} \leq \varepsilon$, and therefore

$$
|\lambda(n)| \leq \frac{\exp \left(n^{\frac{1}{p+1}}\right)}{\varepsilon \exp \left(c n^{\frac{1}{2}}\right)} \leq C \exp (-c \sqrt{n})
$$

for some $C>0$ and all $n \in \mathbf{Z}_{+}$. Thus (vi) $\Longrightarrow(\mathrm{i})$.

Remark 5.2. It was proved by Yanagihara [Y2] that each continuous linear functional $T$ on the Smirnov class $N_{*}$ is of the form

$$
T f=\sum_{n=0}^{\infty} \widehat{f}(n) \lambda(n),
$$

where $(\lambda(n))$ is a multiplier from $N_{*}$ into $H^{\infty}$. This and Theorem 5.1 gives an easy "soft" proof that $T$ is of the form $(\mathrm{LF})$, where $\lambda(n)=O(\exp (-c \sqrt{n}))$ for some $c>0$

\section{Open problems}

Problem 6.1. Find the solid hulls of the Hardy-Orlicz spaces $L H^{p}, p>1$ on the unit disk.

Problem 6.2. Find the solid hulls of the Nevanlinna class $N(D)$ or the Smirnov class $N_{*}(D)$, where $D$ is the unit polydisk or the unit ball in $\mathbf{C}^{n}$.

\section{References}

[D] Duren, P. L.: Theory of $H^{p}$ spaces. - Academic Press, New York and London, 1979.

[DRS] Duren, P.L., B. W. Romberg, and A. L. Shields: Linear functionals on $H^{p}$ with $0<p<1$. - J. Reine Angew. Math. 238, 1969, 32-60.

[JMWX] Jarchow, H., V. Montesinos, K. J. Wirths, and J. XiaO: Duality for some Laree spaces of analytic functions. - Proc. Edinb. Math. Soc. 44, 2001, 571-583.

[JP1] Jevtić, M., and M. Pavlović: On the solid hull of the Hardy space $H^{p}, 0<p<1$. Michigan Math. J. 54, 2006, 439-446.

[JP2] Jevtić, M., and M. Pavlović: On the solid hull of the Hardy-Lorenz space. - Publ. Inst. Math. (Beograd) (N.S.) 85:99, 2009, 55-61.

[K] Kisliakov, S.: Fourier coefficients of boundary values of analytic functions in the disc and bidisc. - Tr. Mat. Inst. Steklova 155, 1981, 77-91.

[MM] MastyŁo, M., and P. Mleczko: Solid hulls of quasi-Banach spaces of analytic functions and interpolation. - Nonlinear Anal. 73, 2010, 84-98.

[P] PrIVAlov, I. I.: Randeigenschaften Analytisher Functionen. - VEB Deutscher Verlag der Wiss. Berlin, 1956.

[S] Shapiro, J. H.: Mackey topologies, reproducing kernels, and diagonal maps on Hardy and Bergman spaces. - Duke Math. J. 43, 1976, 187-202. 
[SS] Shapiro, J. H.,, and A. L. Shields: Unusual topological Properties of the Nevanlinna class. - Amer. J. Math. 97, 1976, 915-936.

[ST] Stoll, M.: Mean growth and Taylor coefficients of some topological algebras of holomorphic functions. - Ann. Polon. Math. 35, 1977, 141-158.

[Y1] Yanagihara, N.: The containing Fréchet space for the Class $N^{+}$. - Duke Math. J. 40, 1973, 93-103.

[Y2] Yanagihara, N.: Multipliers and linear functionals for the class $\mathrm{N}^{+}$. - Trans. Amer. Math. Soc. 180, 1973, 449-461.

[Y3] Yanagihara, N.: Mean growth and Taylor coefficients of some classes of functions. Ann. Polon. Math. 30, 1974, 37-48.

Received 30 January 2014 • Accepted 25 April 2014 